Rev Chil Salud Pública 2012;

Vol 16 (1): 26-31

Artículo original

\section{Determinación de los Índices CPO-D e IHOS en estudiantes de la Universidad Veracruzana, México}

\section{RESUMEN}

Introducción: El conocimiento de la salud bucal de la población constituye una tarea fundamental desde la perspectiva de la salud pública, ya que las enfermedades bucales - tales como la caries y las periodontopatías- son los padecimientos con mayor incidencia y prevalencia alrededor del mundo. El objetivo de esta investigación es determinar el índice de dientes, cariados, perdidos, obturados (CPOD), determinar el índice de higiene oral simplificado (IHOS), de los jóvenes universitarios de nuevo ingreso en el estado de Veracruz, provincia de México. Material y método: se realizó un estudio transversal, descriptivo, observacional, mediante la aplicación del examen de salud integral el cual constó de dos partes; un interrogatorio y una exploración clínica. Resultados: El índice CPOD fue de 13.1. La prevalencia de caries fue de 80.9, perdidos 37.9 y de obturados 47.0. El índice de higiene oral simplificada que se obtuvo fue: el $81.5 \%$ bueno, el $17.4 \%$ regular y el $1.02 \%$ malo. Los alumnos que ingresaron tuvieron un índice CPO muy alto según los estándares de la OMS. Discusión: Los hallazgos en la determinación del índice CPO-D que se registró en este estudio determinan un serio problema de salud pública, Esta situación no sólo es particular en nuestro país, sino que se da la misma tendencia en otros lugares, aspectos que determinan intervenciones necesarias en el ámbito de la salud pública para una mejor calidad de vida de los estudiantes universitarios.

Palabra clave: Higiene bucal, Índice CPO, Índice de higiene oral simplificado

\section{DETERMINING OHI-S AND DMFT IN STUDENTS AT UNIVERSIDAD VERACRUZANA, MÉXICO}

\section{ABSTRACT}

Introduction: Knowledge of the dental health of the population constitutes a fundamental task of public health, since oral diseases- such as cavities and periodontal disease- are the health conditions with highest incidence and prevalence around the world. The objective of this study was to determine the index of decayed, missing or filled teeth (DMFT) and the simplified oral health index (OHI-S) of first year university students in the state of Veracruz, province of Mexico. 
Material y method: A descriptive, cross-sectional, observational study was carried out, applying a comprehensive health exam which consisted of two parts: a questionnaire and a clinical exam. Results: The DMFT index was 13.1. The prevalence of cavities was 80.9 , missing teeth was 37.9 y filled teeth was 47.0 . The OHI-S was the following: $81.5 \%$ good, $17.4 \%$ regular and $1.02 \%$ bad. Students had a very high OHI-S according to WHO standards. Discussion: The findings for the DMFT index constitute a serious public health problem. This situation is not found only in our country. Findings should guide the necessary interventions in order to improve quality of live for university students.

Key words: Oral health, DMFT index, OHI-S index.

\section{INTRODUCCIÓN}

La salud integral como concepto involucra el equilibrio biológico, físico, emocional y social, por tanto la salud bucal es parte integral de la salud general y es esencial para el bienestar y la calidad de vida, en el ser humano. Entre otros aspectos implica estar libre de dolor, tener dientes saludables, poder alimentarse, no tener lesiones en los tejidos y contar con una imagen que estimule el valor de autoestima que proporcione confianza en las relaciones con el medio social; todo esto impacta positivamente en la calidad de vida, sobre todo cuando se inicia una nueva etapa en la formación académica que, sin duda, también afecta los aspectos sociales, culturales y axiológicos.

Actualmente, la sociedad contemporánea otorga un alto valor a la estética física, pero debemos señalar que no sólo esto es importante, ya que si no se cuenta con la salud desde la perspectiva funcional no existirá la armonía entre las estructuras que conforman el aparato estomatognático.

Dentro de los objetivos de salud bucal para el año 2020 recomendados por la Federación Dental Internacional (FDI), la OMS y la Asociación Internacional de Investigación Dental (IADR) proponen retos globales para los encargados de la planeación de programas en salud, en los planos nacional, regional y local; en consecuencia, se ha determinado un máximo de tres dientes cariados para la población escolar y la conservación de todos los dientes en $85 \%$ de los adolescentes de 18 años 1

Entre las principales enfermedades que afectan la cavidad bucal, figuran la caries dental y las enfermedades periodontales ${ }^{2}$. Asimismo la Organización Mundial de la Salud (OMS), reconoce a estas patologías como las de mayor prevalencia, dejando atrás aquellas consideradas como de frecuencia media como las anomalías cráneo-facio-dentales y mal oclusiones ${ }^{3}$, tan solo en nuestro país de acuerdo a los resultados del sistema de Vigilancia Epidemiológica de patologías bucales 2009 (Sivepab) ${ }^{4}$, con información de usuarios que acuden a los servicios de salud odontológicos, el 100\% padece caries dental y $53 \%$ presenta algún grado de enfermedad periodontal, asimismo en la población de 6 a 19 años el índice CPOD promedio fue de 3.8. Las cifras muestran un incremento en la experiencia de caries dental con la edad, encontrándose el mas alto a los 19 años, con un promedio de 7.3 dientes afectados, en este sentido algunos medidores tales como el índice CPOD (sumatoria de los dientes cariados, perdidos y obturados) y el IHOS (índice de higiene oral simplificado), han permito determinar $y$ enfatizar este problema de salud publica cuya reducción, a pesar de los esfuerzos de las entidades de salud en México, ha sido de manera paulatina.

La Organización Mundial de la Salud ${ }^{5-10}$, define el índice CPO-D en dentición permanente a los 12 años como indicador de salud bucal de una población y de acuerdo con su valor establece una escala de gravedad de la afección con cinco niveles:

$$
\begin{aligned}
& \text { Muy bajo } 0.0-1.1 \\
& \text { Bajo } 1.2-2.6 \\
& \text { Moderado } 2.7-4.4 \\
& \text { Alto } 4.5-6.5 \\
& \text { Muy alto }+6.6
\end{aligned}
$$

Esta metodología señala la experiencia de caries tanto presente como pasada, pues considera los dientes con lesiones de caries y con 
tratamientos previamente realizados. Se utiliza para obtener una visión global de cuánto ha sido afectada la dentición por enfermedades dentales. El objetivo de esta investigación es precisamente determinar el índice de higiene oral simplificado (IHOS), de los jóvenes universitarios de nuevo ingreso, con el fin de establecer un diagnóstico e instituir planes de tratamiento en este segmento poblacional.

\section{MATERIAL Y MÉTODO}

Se realizó un estudio transversal, descriptivo, observacional, en la población estudiantil universitaria de nuevo ingreso, se determinó un muestreo intencional o de conveniencia constituido por 195 estudiantes de nuevo ingreso del ciclo escolar agosto 2010-febrero 2011, de tres licenciaturas de la Universidad Veracruzana campus Minatitlán; (65 estudiantes de la Facultad de Odontología, 65 estudiantes de la Facultad de Medicina y 65 estudiantes de la Facultad de Enfermería).

La recolección de datos se hizo en la clínica II de la Facultad de Odontología a través de la aplicación de un instrumento: "Examen de Salud Integral”, diseñado en la clínica de la universidad que consta de dos apartados, uno de interrogatorio y otro de exploración.

Las variables de estudio comprendieron la edad, género e higiene bucal, en donde se preguntó aspectos relacionados con el número de veces de cepillado dental, uso de la técnica correcta de cepillado, profilaxis, presencia de gingivorragia espontánea y mecánica, tratamientos ortodónticos actual y anterior, dolor en región de terceros molares. En el apartado de exploración clínica se determinó el índice CPO-D, e IHOS (índice de higiene oral simplificado).

Los sujetos de estudio fueron revisados por el equipo de investigación conformado por dos asistentes, los cuales fueron instruidos sobre la metodología para la recolección de los datos. En cuanto al apartado del interrogatorio se realizó una prueba piloto, la cual permitió ajustes del instrumento; con relación al proceso de exploración los odontólogos asistentes fueron capacitados para el levantamiento de los Índices de CPO-D (Klein y Palmer): el índice CPOD describe numéricamente los resultados de la

afectación por caries en los dientes permanentes de un grupo poblacional.

De acuerdo a la OMS los niveles de severidad en prevalencia de caries se determinan en:
$0.0-1.1$ Muy bajo
$1.2-2.6$ Bajo
2.7 - 4.4 Moderado
$4.5-6.5$ Alto
6.6 Muy alto

Para el levantamiento del Índice Simplificado de Higiene Bucal (IHO-S) (Green y Ver millón), se examinaron 6 piezas dentarias según la metodología de este índice: $1^{\circ}$ molar permanente superior derecho (superficie vestibular); incisivo central superior permanente derecho (superficie vestibular); $1^{\circ}$ molar permanente superior izquierdo (superficie vestibular), $1^{\circ}$ molar permanente inferior izquierdo (superficie lingual); incisivo central inferior permanente izquierdo(superficie vestibular) y $1^{\circ}$ molar permanente inferior derecho (superficie lingual). Asimismo, el índice IHO-S consta de dos componentes: el índice de residuos simplificado (DIS) y el índice de cálculo (Cl-S), cada componente se evalúa en una escala de 0 a 3.

Los criterios para medir el componente de residuos (DI-S) de higiene oral simplificada (IHO-S) fueron los siguientes:

0 - No hay residuos o manchas.

1- Los residuos o placa no cubre más de un tercio de la superficie dentaria.

2- Los residuos o placa cubren más de un tercio de la superficie pero no más de dos tercios de la superficie dentaria expuesta.

3- Los residuos blandos cubren más de 2 tercios de la superficie dentaria expuesta.

Para obtener el índice individual de IHO-S por individuo se requiere sumar la puntuación para cada diente señalado y dividirla entre el número de superficies analizadas, una vez ya establecido, se procede a determinar el grado clínico de higiene bucal:

Excelente: 0,0

Bueno: 0,1 - 1,2

Regular: 1,3 - 3,0

Malo: 3,1 - 6,0 
Los datos obtenidos fueron tabulados y graficados de acuerdo a cada índice y a las variables de estudios para ser analizadas en los programas estadísticos MS Excel y el SPSS Ver.19.0

\section{RESULTADOS}

Después de aplicar la encuesta y realizar el examen exploratorio en la totalidad de los estudiantes que participaron en el estudio, se determinaron aspectos importantes desde el punto de vista de la salud bucal.

La edad predominante en la población de estudio de las tres licenciaturas estaba conformada entre los 18 años constituyendo el $46.6 \%$, siendo el de menor representatividad los de 21 años con el $5.1 \%$, considerando que no existe un límite de admisión a este nivel superior de estudio. Asimismo, el género femenino fue el sobresaliente estableciendo el $70 \%$ de la población estudiada. Algunos de los datos importantes en esta investigación se centran en el número de veces que los estudiantes realizan el cepillado de los órganos dentarios (Figura 1), dado que, según la literatura odontológica, este aspecto se encuentra relacionado con la presencia de patologías y alteraciones bucales.

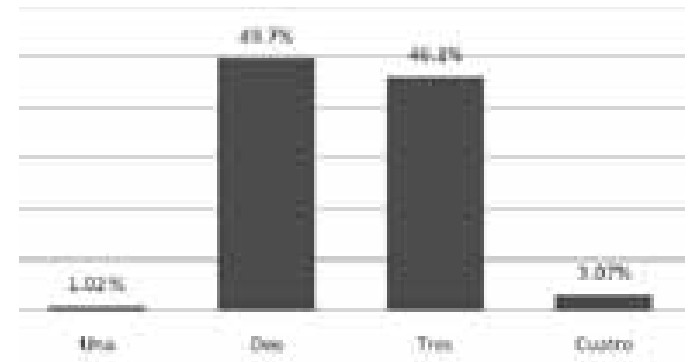

Figura 1. Distribución porcentual sobre el número de veces que los encuestados realizan el cepillado dental durante el día.

Durante la exploración clínica en el 89.0 $\%$ no se encontró gingivorragia espontánea; sin embargo, el $77 \%$ de la población estudiada sí presento gingivorragia al realizar la estimulación mecánica, asimismo, se pudo observar que el $11.0 \%$ refiere haber presentado hemorragia de las encías sin ningún motivo aparente.

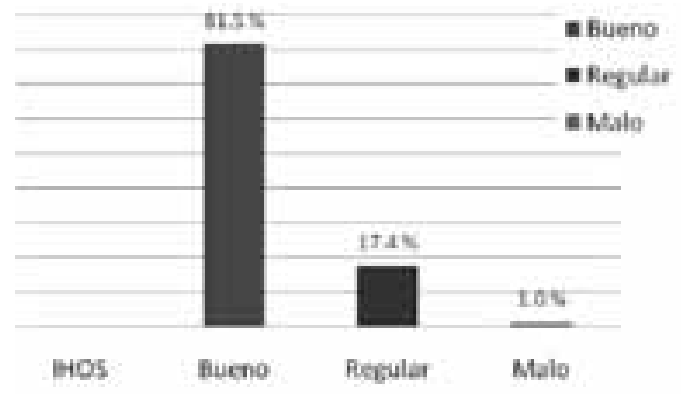

Figura 2. Distribución porcentual sobre la escala obtenida en la evaluación del índice oral simplificado

El resultado de la evaluación del IHOS, determinó que los estudiantes que ingresan a la universidad en el área de ciencias de la salud se sitúan en una escala de ponderación de (81.5), el cual según la escala es considerado como aceptable (Figura 2).

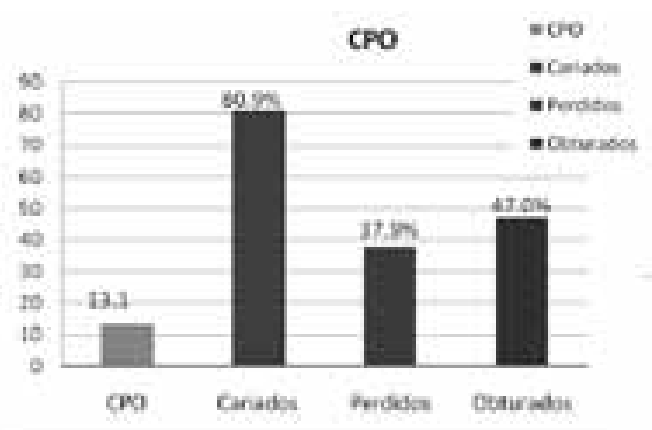

Figura 3. Índice CPO obtenido y distribución porcentual del estado de salud oral en la población estudiantil estudiada.

Sin embargo el índice CPO-D obtenido fue muy alto, 13.1 sobrepasando la escala establecida por la $\mathrm{OMS}^{5}$, el cual considera el intervalo de 6.6 como muy alto. Este indicador determinó la prevalencia de caries en los estudiantes en un $80.9 \%$, considerándose también que casi la mitad de los órganos dentarios revisados han sido tratados por este padecimiento $(47.0 \%)$, y el $37.9 \%$ han sido extraídos, aspectos que hay que considerar pues el área estudiada involucra a jóvenes que ingresan al área de la salud quienes necesitan mantener niveles bajos de alteraciones y patología bucales. 


\section{DISCUSIÓN}

Los datos obtenidos y analizados en el presente estudio indican que la situación de la salud bucal de los estudiantes que ingresaron a la Universidad Veracruzana campus Minatitlán en el ciclo escolar agosto 2010-febrero 2011, presentaron problemas de salud, haciéndose relevante los altos índices de caries dental, aspecto que constituye un problema de salud pública, ya que a pesar de las metas establecidas en los programas de salud en nuestro país, aún los índices de caries y enfermedad periodontal son altos $^{6}$

Esta situación no solo es particular en nuestro país, sino que se da la misma tendencia en otros lugares. En estudios similares como en Honduras ${ }^{7}$ se observó un CPO-D de 14.6, un poco más elevado que en los hallazgos de nuestro estudio.

En el ámbito nacional, en un estudio realizado por la Universidad Autónoma de Nayarit ${ }^{8}$, en estudiantes de la licenciatura de cirujano dentista el resultado del índice CPOD fue de 8.45, lo que sitúa este aspecto en una escala crítica con relación a los índices de salud bucal aceptables.

En contraste, en un estudio similar en nuestro país en la licenciatura de cirujanos dentistas FES Iztacala ${ }^{9}$, se obtuvo que el CPO fue de 3.8 muy por debajo de nuestros resultados, debiendo destacar que el estudio fue realizado en alumnos, quienes ya cursaban la licenciatura y poseían algún grado de educación preventiva dado a su perfil de formación

Con relación al IHOS, en el estudio realizado en la población hondureña ${ }^{7}$ se encontró que el $72.1 \%$ tenía un índice de higiene oral aceptable; en comparación, en nuestro estudio el $81.5 \%$ de los encuestados presentó una buena higiene bucal, mientras que el $17.4 \%$ una higiene bucal regular, y sólo el $1.02 \%$ de los estudiantes tuvieron una higiene bucal deficiente.

Los resultados permiten establecer que los estudiantes universitarios de nuevo ingreso del campus Minatitlán, presentan un alto índice de afectación de la salud bucal, principalmente de caries. Es necesario establecer estrategias de prevención y rehabilitación de la salud bucal de los jóvenes dado que en este contexto algunas cifras no son alentadoras, pues la OMS espera que la incidencia de caries dental aumente en muchos países en vías de desarrollo particularmente como resultado de un consumo cada vez mayor de azúcares y la exposición inadecuada a los floruros.

Asimismo, es necesario implementar estrategias para la prevención y promoción de la salud bucal a los estudiantes en edad universitaria. Si bien existen programas en el ámbito nacional para los escolares de nivel primaria y preescolar, también constituye una necesidad incluir a este segmento de la población para la reorientación de los hábitos dietéticos e higiénicos que permitan crear una cultura referente a la salud bucal.

\section{REFERENCIAS}

1. Hobdell M, Petersen PE, Clarkson J. Goals for oral health 2020. Int Dent J. 2003;53:285-288.

2. Harris N, García-Godoy F. Odontología preventiva primaria. México: El Manual Moderno; 2008.

3. Norma Oficial Mexicana para la prevención y control Prevención y control de enfermedades bucales: modificación a la Norma Oficial Mexicana, NOM-013SSA2-1994 [en línea] (6 de enero de 1995), [ citado en septiembre de 2011]. Disponible en: http://www.salud. gob.mx/unidades/cdi/nom/013ssa24.html

4. México. Secretaría de Salud, México. Centro Nacional de Vigilancia Epidemiológica y Control de Enfermedades. Resultados Sistema de Vigilancia Epidemiológica de las Patologías Bucales 2009 [en línea]. México: Secretaría de Salud; 2010 [citado en septiembre de 2011] Disponible en: http://cenavece. salud.gob.mx/opencms/opencms/programas/interior/ saludbucal/descargas/zip/sivepab2009.zip

5. Organización Mundial de la Salud [en línea]. Ginebra: OMS; c2012 [citado en septiembre de 2011]. La OMS publica un nuevo informe sobre el problema mundial de las enfermedades bucodentales. Disponible en http://www.who.int/mediacentre/news/releases/2004/ pr15/es/

6. México. Subsecretaría de Prevención y Promoción de la Salud. Programa de acción especifico 2007-2012 [en línea]. $1^{\text {a }}$ ed. México: SPPS; 2008 [citado en septiembre de 2011]. Disponible en: http://www.spps.gob. $\mathrm{mx} /$ images/stories/SPPS/Docs/proy_prog/25._ve.pdf

7. Arévalo SJ, Félix - Rivera M, Rivera-Sánchez F. Situación de la salud bucal de la población universitaria hondureña. Rev Méd Hondureña. 2005;73:161-165.

8. Aguilar-Orozco N, Navarrete-Ayón K, Robles-Romero D, Aguilar-Orozco SH, Rojas-García A. Dientes sanos, cariados, perdidos y obturados en los estudiantes de la Unidad Académica de Odontología de la Universi- 
dad Autónoma de Nayarit. Revista Odontológica Latinoamericana. 2009;1(2):27-32.

9. Esquivel HR, Jiménez FJ .Perfil Epidemiológico de salud bucodental de la Fes Iztacala. Revista Odontológica Mexicana. 2007;11:25-32

10. Vergara -Arriata K, Osorio- Castillo M, Paternina- Mo- rales J. Prevalencia de caries y enfermedad periodontal en estudiantes del programa de Odontología de la Universidad de Cartagena en el segundo periodo. Colombia: Universidad de Cartagena; 2007.

Recibido: 25 de noviembre de 2011 Aprobado: 7 de marzo de 2012 